\title{
Visualisation in environmental sciences
}

\author{
Karsten Rink • Gerik Scheuermann • \\ Olaf Kolditz
}

Received: 27 August 2014/ Accepted: 30 September 2014/Published online: 14 October 2014

(C) Springer-Verlag Berlin Heidelberg 2014

In recent years, the amount of environmental data that is measured, collected and analysed has risen in staggering amounts. The reason for this is largely the desire and need of both science and politics to better understand the reasons, effects and possible consequences of climate change. This includes topics as diverse as the behaviour of groundwater regimes in arid regions, the migration of animal species, or the wide range of ecosystem services. In addition, this trend is reinforced by observation methods getting more and more refined: satellites can measure data in the sub-metre range, eddy covariance systems analyse high-frequency atmospheric data such as wind, heat or gas fluxes and weather surveillance radar creates high-resolution data of precipitation parameters every few minutes, to name just a few examples.

Vital insight is gained from correlating such data or simulating certain processes based on the collected information. Examples range from devising water management schemes for arid and humid regions, feasible plans for the transition from fossil to renewable energy sources, finding adequate storage sites for nuclear waste, or the effects of chemicals discharged into the atmosphere by industry or into the water in the form of agricultural fertilisers.

\footnotetext{
K. Rink $(\square) \cdot$ O. Kolditz

Department of Environmental Informatics, Helmholtz Centre for Environmental Research, Leipzig, Germany

e-mail: karsten.rink@ufz.de

G. Scheuermann

Institut für Informatik, Universität Leipzig, Leipzig, Germany

O. Kolditz

Applied Environmental System Analysis, Technische Universität Dresden, Dresden, Germany
}

Developing effective visualisation techniques is the key to improving the understanding of complex data sets and communicate findings to the public in general and stakeholders in particular. This process requires collaboration of researchers from environmental sciences (geology, meteorology, hydrology, etc.) and computer scientists with experience in handling and visualising large amounts of heterogenous data. While this has previously worked well in other domains, e.g. for the visualisation of complex medical data (Preim and Botha 2013; Deserno 2011), in environmental sciences visualisation is often viewed as an addendum. Once the field work and simulations are done, results are visualised to show the results to other interested parties. The possibilities for visualisation in assisting scientists in their research for verification, integration and analysis of the data are often neglected outside of the established domains of timeseries analysis or cartographic data in geographic information systems. In addition, visualisation is an excellent means of communication between researchers from different disciplines and facilitating support for basic workflows such as model creation.

This thematic issue includes expanded and new articles based on presentations of the workshop "Visualisation in Environmental Sciences", which took place as a co-located event of EuroVis 2013, the EuroGraphics conference on visualisation. Contributions included applications from the domains of biodiversity (Slingsby and van Loon 2013), migration ecology (Kölzsch et al. 2013), ecosystem restoration (Eligehausen et al. 2013), flood analysis (Schlegel et al. 2013), 3D geological modelling (Lidal et al. 2013) and urban climate simulation (Röber et al. 2013). The intent is to give readers a notion about the multitude of possibilities for visualisation in the scope of environmental sciences. 
George et al. (2014) propose a framework for the analysis of sea level rise due to climate change. An interactive $2 \mathrm{D}$ visualisation of the multiple aspects of the data allows the exploration of simulated scenarios and setting of transects to assess information about hydrodynamic flux. The focus of their study was a fast and intuitive visualisation of the data to support the analysis of hydro- and morphodynamics of estuaries in the scope of flood management research. The usability of the framework was tested in close collaboration with oceanography experts to ensure its usability in future studies.

The article by Helbig et al. (2014) is also concerned with climate change. The authors propose a number of $3 \mathrm{D}$ visualisation techniques to understand and validate the results of complex climate simulations. By assigning certain types of representations to various phenomena, the information from a dense multi-parameter data set becomes intuitively understandable. With parameter variation and interaction, regions of interest can be assessed and evaluated. To determine the quality of simulation results, observed and simulated data can be compared and differences highlighted. Using the framework in a virtual reality environment provides a basis for interdisciplinary discussions.

Häb et al. (2014) present framework for the feature extraction from multi-temporal thermograph imagery of certain construction elements or surfaces. In combination with other data, this information supports data analysis in the scope of urban micro-climate research. The proposed software first employs a skilful combination of established image processing techniques to detect and register features from image sequences. Identified candidates are then extracted using explicit active contours. Using interpolation schemes, animations of features over time can be visualised. Also, the temporal behaviour of such features can be analysed using directed graphs in which nodes encode properties of the detected features. Extraction is then successfully demonstrated based on low-resolution image sequences acquired using a hand-held scanner.

Naumov et al. (2014) approach the problem of visualisation of complex geometries in fluid dynamic simulations. Based on pore space examples, they propose a technique for data exploration, especially in virtual reality environments, that allows the assessment of simulation data while still retaining a concept of the underlying geometry. A detailed analysis on the use of specific shaders and rendering algorithms presents an in-depth view on the use of custom-made computer graphics techniques in hydrodynamic process simulation.

Using visualisation in an entirely different context, Pham et al. (2014) employ information visualisation techniques for diversity analysis. The authors focus on the development of a framework using common characteristics and goals for multivariate object analysis. Adequate representation techniques for assessing certain types of data are suggested and the importance of visualisation as a communication tool between researchers from different disciplines is emphasised.

The second half of the contributions in this thematic issue discusses ways in which visualisation can support 3D geological modelling to solve different kinds of challenges.

Walther et al. (2014) discuss the remediation potential of a salinised coastal aquifer in the Middle East. Starting with a comparison of numerical models with laboratory experiments, potential problems are identified and conclusions are applied to a regional scale model. Visualisation techniques are also able to reveal potential ways for improving a model based on scarce data. Based on different parameterisations, the complex flow processes within the model region are then explored in an interactive 3D scene using a number of filters to highlight interesting aspects within the data.

Ling and Chen (2014) employ the Environmental Visualisation System for data interpretation and exploration of hydrogeological processes. Large amounts of environmental information within test sites are synthesised and analysed, focussing on contaminant distribution and remediation. Similar to the previous paper, visualisation is not only used for understanding and interpretation of the data but also to facilitate remedial and/or monitoring designs.

Liu et al. (2014) make use of 3D visualisation for an indepth analysis of a large test field in the Three Gorges Reservoir Region in China that is prone to substantial landslides. Their model is to further the knowledge of landslide research in general based on this particular example. The data for this model are derived from an extensive database including not only GIS data but also statistical and physical parameters that can be incorporated into the 3D visualisation along with artificial tunnels for an inside view of the model.

Rink et al. (2014) propose an open-source framework for data exploration, integration and model setup to simulate hydrological phenomena. A number of common problem classes are discussed regarding the handling of missing or conflicting information. In addition, general techniques are suggested to present complex data collections from case studies to an outside audience.

Schimpf and Gossel (2014) propose a modular opensource framework to explore geological data. By supporting a large number of national and international standards for geological information as well as many established file formats, existing information is easily integrated and can be examined in $2 \mathrm{D}$ or $3 \mathrm{D}$ in combination with relevant parameters. In addition, it is possible to create virtual boreholes and cross sections from existing 3D models. 
Finally, a technical paper by Bilke et al. (2014) illustrates the workflow and logistics for presenting data from a wide range of environmental case studies in an immersive virtual reality environment. It touches on the technical requirements, advances in recent years (such as the rise of head-mounted displays) and highlights the need for interdisciplinary cooperation for the creation of environmental studies.

The articles presented here highlight a number of benefits of visualisation for the exploration and analysis of complex environmental data and models. They also underscore how much interdisciplinary collaboration improves the understanding of the data itself as well as the processes and techniques necessary for an adequate presentation. We hope that this small selection of examples provides an incentive to other researchers to make use of visualisation techniques in their field of work for better insight into their data and processes as well as a tool for communication with others.

Acknowledgments The guest editors would like to thank the contributing authors who present a detailed view of the possibilities of visualisation in environmental sciences both from a environmental and from a computer science point of view. Many thanks also to the reviewers for their comments to improve the quality of the articles presented here. Further acknowledgements to particular project funding are referred to in the individual papers of this thematic issue.

\section{References}

Bilke L, Fischer T, Helbig C, Krawczyk C, Nagel T, Naumov D, Paulick S, Rink K, Sachse A, Schelenz S, Walther M, Watanabe N, Zehner B, Ziesch J, Kolditz O (2014) TESSIN VISLablaboratory for scientific visualization. Environ Earth Sci (this issue)

Deserno TM (ed) (2011) Biomedical image processing. Springer, Berlin

Eligehausen J, Riecken L, Borchardt D (2013) BlendGIS: an open source tool for $3 \mathrm{~d}$ and $4 \mathrm{~d}$ visualization of ecology related spatial data. In: Kolditz O, Rink K, Scheuermann G (eds) Proceedings of workshop on visualisation in environmental sciences (EnvirVis), pp 1-5. Eurographics Association. doi:10.2312/PE.Envir Vis.EnvirVis13.001-005

George RLSF, Robins PE, Davies AG, Ritsos PD, Roberts JC (2014) Interactive visual analytics of hydrodynamic flux for the coastal zone. Environ Earth Sci. doi:10.1007/s12665-014-3283-9

Häb K, Feige NH, Hüttenberger LS, Middel AC, Hagen H (2014) Visualizing the temporal development of thermo-radiative features on ground-based thermographs. Environ Earth Sci. doi:10.1007/s12665-014-3472-6

Helbig C, Bauer HS, Rink K, Wulfmeyer V, Frank M, Kolditz O (2014) Concept and workflow for $3 d$ visualization of atmospheric data in a virtual reality environment for analytical approaches. Environ Earth Sci. doi:10.1007/s12665-014-3136-6

Kölzsch A, Slingsby A, Wood J, Nolet BA, Dykes J (2013) Visualisation design for representing bird migration tracks in time and space. In: Kolditz O, Rink K, Scheuermann G (eds) Proceedings of workshop on visualisation in environmental sciences (EnvirVis), pp 25-29. Eurographics Association. doi:10.2312/PE.EnvirVis.EnvirVis13.025-029

Lidal EM, Patel D, Bendiksen M, Langeland T, Viola I (2013) Rapid sketch-based 3D modeling of geology. In: Kolditz O, Rink K, Scheuermann G (eds) Proceedings of workshop on visualisation in environmental sciences (EnvirVis), pp 31-35. Eurographics Association. doi:10.2312/PE.EnvirVis.EnvirVis13.031-035

Ling M, Chen J (2014) Environmental visualization: Applications to site characterization, remedial programs, and litigation support. Environ Earth Sci. doi:10.1007/s12665-014-3220-y

Liu J, Tang H, Zhang J, Shi T (2014) "glass landslide"-the 3d visualization makes study of landslide transparent and virtualized. Environ Earth Sci. doi:10.1007/s12665-014-3183-z

Naumov D, Bilke L, Kolditz O (2014) Rendering technique of multilayered domain boundaries and its application to fluid flow in porous media visualizations. Environ Earth Sci. doi:10.1007/ s12665-014-3445-9

Pham T, Jones J, Metoyer R, Colwell F (2014) Toward exploratory analysis of diversity unified across fields of study. Environ Earth Sci. doi:10.1007/s12665-014-3365-8

Preim B, Botha C (2013) Visual computing for medicine, 2nd edn. Morgan Kaufmann, Burlington

Rink K, Bilke L, Kolditz O (2014) Visualisation strategies for environmental modelling data. Environ Earth Sci. doi:10.1007/ s12665-013-2970-2

Röber N, Salim M, Gierisch A, Böttinger M, Schlünzen H (2013) Visualization of urban micro-climate simulations. In: Kolditz O, Rink K, Scheuermann G (eds) Proceedings of workshop on visualisation in environmental sciences (EnvirVis), pp 53-57. Eurographics Association. doi:10.2312/PE.EnvirVis.EnvirVis13. 053-057

Schimpf L, Gossel W (2014) From registration to visualization of geological data with mo2geo: applications of the fieldmodule, interpolation methods, and computer visualization. Environ Earth Sci. doi:10.1007/s12665-014-3114-z

Schlegel S, Böttinger M, Hlawitschka M, Scheuermann G (2013) Determining and visualizing potential sources of floods. In: Kolditz O, Rink K, Scheuermann G (eds) Proceedings of workshop on visualisation in environmental sciences (EnvirVis), pp 65-69. Eurographics Association. doi:10.2312/PE.EnvirVis. EnvirVis13.065-069

Slingsby A, van Loon E (2013) Visual analytics for exploring changes in biodiversity. In: Kolditz O, Rink K, Scheuermann G (eds.) Proceedings of workshop on visualisation in environmental sciences (EnvirVis), pp 71-75. Eurographics Association. doi: 10.2312/PE.EnvirVis.EnvirVis 13.071-075

Walther M, Bilke L, Delfs JO, Graf T, Grundmann J, Kolditz O, Liedl $R$ (2014) Assessing the saltwater remediation potential of a three-dimensional, heterogeneous, coastal aquifer system model verification, application and visualization for transient densitydriven seawater intrusion. Environ Earth Sci. doi:10.1007/ s12665-014-3253-2 